\title{
BOUND STATES OF FERMIONS ON 2D LATTICE IN A DILUTE LIMIT
}

\author{
M. BĄ $\mathrm{K}^{*}$ AND R. MICNAS ${ }^{\dagger}$ \\ Institute of Physics A. Mickiewicz University \\ Umultowska 85, 61-614 Poznań, Poland
}

We examine extended bound states in a dilute limit of the extended Hubbard model on the two-dimensional square lattice. By solving exactly the two-body problem we have determined the binding energies, mobilities, and dispersion curves across the Brillouin zone for bound states of various symmetries. It turns out that the $d$-wave pairing is strongly favored by the nnn hopping and the intersite local pairs can have small effective masses, even in the case of strong binding. We have also found a possibility of extended $s-d_{x^{2}-y^{2}}$ mixing of the bound states.

PACS numbers: 74.20.-z, 03.65.Ge, 71.10.Fd

\section{Introduction}

The case of a bound fermion pair in an empty lattice is one of few rigorous results in the theory of superconductivity. It can be regarded as a limit of both BCS theory and local pairing theory. In this case we can solve exactly the two-body Schrödinger equation $[1,2]$. The results give an insight into the behavior of pairs of different symmetries (important, as the pairing symmetry in many HTS is believed to be of the $d_{x^{2}-y^{2}}$, hereafter $d$, type). The critical values for binding of the intersite extended $s$-type $\left(s^{*}\right)$ pairs are also critical values for the superconductivity in $2 \mathrm{D}$ systems with low electron density. In this paper we present chosen results for the binding energies, mobilities, and dispersion of singlet bound pairs (BS) in the extended Hubbard model.

\section{The method}

We examine BS in the extended Hubbard model on a 2D square lattice

$$
\begin{aligned}
& H=H_{0}+H_{1}, \quad H_{0}=\sum_{i j \sigma} t_{i j} c_{i \sigma}^{\dagger} c_{j \sigma}, \\
& H_{1}=\sum_{i j} g_{i j}^{\mathrm{S}} S_{i j}^{\dagger} S_{i j}+\sum_{\mu i j} g_{i j}^{\mathrm{T}} T_{\mu i j}^{\dagger} T_{\mu i j}
\end{aligned}
$$


where $i, j$ denote the lattice sites, $c_{i \sigma}$ is an electron annihilation operator, $S_{i j}=$ $(1 / 2)\left(c_{i \uparrow} c_{j \downarrow}+c_{j \uparrow} c_{i \downarrow}\right)$ is a singlet electron pair annihilation operator, $T_{\mu}$ are triplet pairs operators: $T_{0}=(1 / 2)\left(c_{i \uparrow} c_{j \downarrow}-c_{j \uparrow} c_{i \downarrow}\right), T_{+}=c_{i \uparrow} c_{j \uparrow} / \sqrt{2}, T_{-}=c_{i \downarrow} c_{j \downarrow} / \sqrt{2}$, $g_{i j}$ is an interaction between the electrons on the lattice sites $i$ and $j: g^{\mathrm{S}}$ - singlet and $g^{\mathrm{T}}$ - triplet one. In the standard notation $g_{i i}=U, g_{i j}=W_{1}, t_{i j}=t_{1}$ for $\mathrm{nn}$ sites and $g_{i j}=W_{2}, t_{i j}=t_{2}$ for $i, j$ being the nnn. In the following we will only consider the singlet pairing channel.

The Schrödinger equation for a two-particle problem is

$$
\left(H_{0}+H_{1}\right)|\boldsymbol{P}, \psi\rangle=E|\boldsymbol{P}, \psi\rangle,
$$

where $|\boldsymbol{P}, \psi\rangle$ is a wave function of a pair with a center-of-mass momentum $\boldsymbol{P} .|\boldsymbol{P}, \psi\rangle$ is expressed as a linear combination of functions $|\boldsymbol{P}, \boldsymbol{p}\rangle$ with relative momentum $p$, which are the eigenfunctions of $H_{0}$ [1]

$$
|\boldsymbol{P}, \psi\rangle=\sum_{\boldsymbol{p}} F(p)|\boldsymbol{P}, p\rangle .
$$

Solving the equation for coefficients $F(p)$ we obtain the pair wave function $\psi(i)$

$$
\psi(i)=\sum_{j} G(E, p, i, j) g_{i j} \psi(j),
$$

where

$$
G(E, \boldsymbol{P}, i, j)=\frac{1}{N} \sum_{\boldsymbol{p}} \frac{\psi(i \mid \boldsymbol{p}) \psi^{*}(j \mid \boldsymbol{p})}{E-E_{\boldsymbol{P} \boldsymbol{p}}}
$$

is the Green function, $\psi(i \mid p)$ are the plane waves and $E_{\boldsymbol{P p}}=-4 t_{1} \times$ $\left[\cos \left(P_{x} / 2+p_{x}\right)+\cos \left(P_{y} / 2-p_{y}\right)\right]-8 t_{2} \cos \left(P_{x} / 2+p_{x}\right) \cos \left(P_{y} / 2-p_{y}\right)$ is the energy of two free electrons (lattice constant is set to 1 ). The equation for $\psi$, on the sites where the interaction $g$ is present, in the matrix form is: $[1-\mathcal{G}(E, \boldsymbol{P}) g] \psi=0$. For other sites we have to use Eq. (4). The energy eigenvalues are calculated from the determinant: $\operatorname{det}[1-\mathcal{G}(E, P) g]=0$.

Transforming to the reciprocal space and making use of the symmetry properties, the wave function can be divided into five parts transforming according to the irreducible representations of the point symmetry group $\left(C_{4 v}\right)$ of the $2 \mathrm{D}$ lattice: $\psi_{0}=1\left(A_{1}, s\right.$-wave $), \psi_{1}(\boldsymbol{p})=\cos \left(p_{x}\right)+\cos \left(p_{y}\right)\left(A_{1}, s^{*}\right), \psi_{2}(p)=\cos \left(p_{x}\right)-$ $\cos \left(p_{y}\right)\left(B_{1}, d_{x^{2}-y^{2}}\right), \psi_{3}(p)=2 \cos \left(p_{x}\right) \cos \left(p_{y}\right)\left(A_{1}, s^{*}\right), \psi_{4}(p)=2 \sin \left(p_{x}\right) \sin \left(p_{y}\right)$ $\left(B_{2}, d_{x y}\right)$. The eigenvalue equation is separated into three independent parts, describing different types of pairing

$$
\operatorname{det}\left(\begin{array}{ccc}
1-G_{00} U & -G_{01} U & -G_{03} U \\
-G_{01} W_{1} & 1-G_{11} W_{1} & -G_{13} W_{1} \\
-G_{03} W_{2} & -G_{13} W_{2} & 1-G_{33} W_{2}
\end{array}\right)=0
$$

for $s^{*}$-type, $\dot{1}+\left|W_{1}\right| G_{22}=0$ for $d_{x^{2}-y^{2}}$-type, and $1+\left|W_{2}\right| G_{44}=0$ for $d_{x y}$-type;

$$
G_{n m}(E, P)=\frac{1}{N} \sum_{\boldsymbol{p}} \frac{\psi_{n}(\boldsymbol{p}) \psi_{m}(\boldsymbol{p})}{E-E_{\boldsymbol{P} \boldsymbol{p}}}
$$

The relative pair mobility is evaluated as

$$
Z=\frac{2 m_{f}}{m^{*}}
$$


where $m_{f}=\hbar^{2} / 2\left(t_{1}+2 t_{2}\right)$ is the mass of a free electron and the effective mass $m^{*}$ of a pair is calculated from the curvature of dispersion curves around the $\Gamma$ point, where the energy minimum is.

\section{Results}

The binding energies and mobilities of bound pairs of different symmetries for nn hopping are shown in Fig. 1. Pairs with the largest binding energy and no threshold to bind are of the $s^{*}$-type for $U=0$. Inclusion of repulsive on-site or intersite interaction decreases the binding energy and a threshold appears, which for $U=\infty$ is $\left|W_{1 c} / 8 t_{1}\right|=0.25$. Pairs of the $d$-type are of the smallest binding energy and the largest critical value: $\left|W_{1 c} / 8 t_{1}\right|=0.915$ for $d_{x^{2}-y^{2}}$ and $\left|W_{2 c} / 8 t_{1}\right|=$ 0.827 for $d_{x y}$ (not shown) pairing. Let us note that neither $U$ nor $W_{2}$ interactions influence pairs of $d_{x^{2}-y^{2}}$ symmetry.

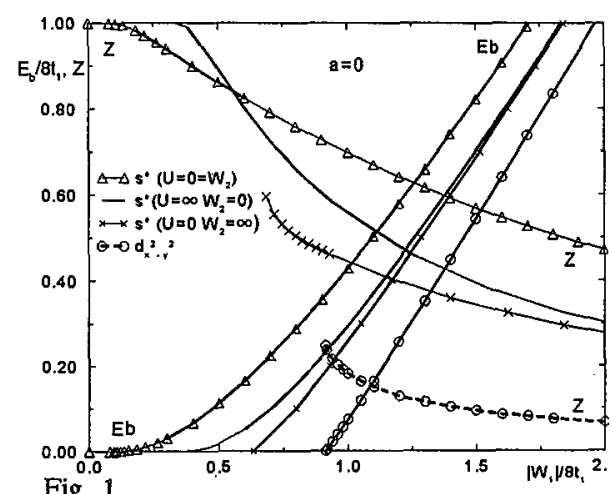

Fig. 1

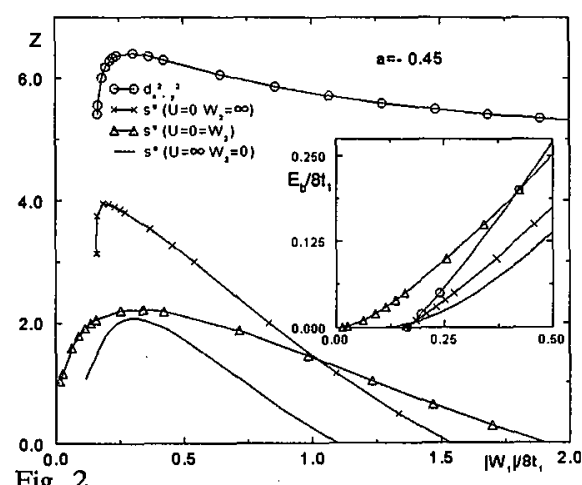

Fig. 2

Fig. 1. Binding energies $\left(E_{b}\right)$ and mobilities $(Z)$ for $t_{2}=0$, for different types of pairing.

Fig. 2. Binding energies and mobilities for $t_{2} / t_{1}=-0.45$, for different types of pairing.

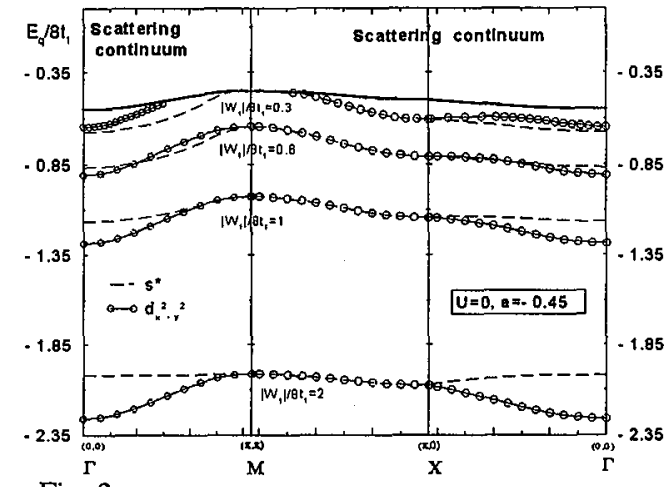

Fig. 3

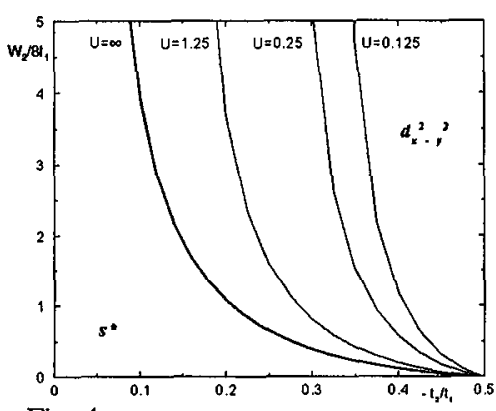

Fig. 4

Fig. 3. Dispersion curves for $s^{*}$ - and $d$-type pairing in $\mathrm{Bz}$ for $\left|W_{1} / 8 t_{1}\right|=0.3,0.6,1,2$ (from top), for $U=0, W_{2}=0$, and $a=-0.45$.

Fig. 4. Stability diagram for shallow $s^{*}$ and $d$ bound states. $U$ in $8 t_{1}$ units. 
The nnn hopping $t_{2}$ (of the opposite sign to $t_{1}$ ) enhances both mobilities and binding energies of pairs of all symmetries. The rate of increase is the largest for $d$-type pairs. Starting from $|a| \approx 0.3\left(a \equiv t_{2} / t_{1}\right)$ the pair mobilities can become larger than 1 and even strongly bound intersite pair can move easily without breaking its bond. For $\left|W_{1}\right| \rightarrow \infty, Z \rightarrow \pm|a| /(1+2 a)$ with $+(-)$ for $d\left(s^{*}\right)$-wave pairs [1]. The plots for $a=-0.45$ (the value corresponding to $\mathrm{YBaCuO}$ ) are shown in Fig. 2, where $d$-type pairs mobility reaches the value $\sim 6$. Also $s^{*}$-wave pairs mobilities are larger than 1, at least in a certain parameter range. They become negative for $\left|W_{1}\right|$ large enough, which is due to moving the $s$-wave dispersion curves minima towards the boundary of the Brillouin zone $(\mathrm{Bz})$ with increasing $\left|W_{1}\right|$. In the inset the binding energies are shown. The $d$ pairs binding energy increases considerably relative to that of pairs of other symmetries, but a threshold still exists for this pairing $\left(\left|W_{1 \mathrm{c}}\right| / 8 t_{1}=0.164\right)$. It is interesting that for small $\left|W_{1}\right|$ the binding energies of $s^{*}$ pairs with $U=0$ are larger than that of $d$ pairs, despite the fact that the $s^{*}$ pairs mobility is smaller than $d$-type one in the whole range of intersite interactions.

The pair dispersion curves are shown in Fig. 3. We can observe moving of $s^{*}$-type pairs minima with increasing $\left|W_{1}\right|$ as was described above. For medium intersite interactions a mixing of the $s^{*}-d$ states occurs. Such a mixing also appears for all $\left|W_{1}\right|$ on the $\mathrm{Bz}$ boundaries, along the lines $M X, M Y$. For small values of the intersite attraction we notice that the shallow $d$ states (with small binding energies) seem to enter the scattering continuum. It is probable that they turn into the resonance states with a finite lifetime. We were unable to obtain real solutions for the binding energies near the corner of the $\mathrm{Bz}$.

In Fig. 4 the $s^{*}-d$ stability diagram for shallow bound states is shown. Above the plotted line the $s^{*}$-type pairs (and $d$ below) cannot exist due to a critical value of $W_{1}$ for binding for a finite $U$. The diagram implies that both increasing $W_{2}$ and nnn hopping act in favor of $d$-type pairing. A peculiar situation occurs for $a=-0.5$ and $W_{2}=0$, where both $s^{*}$ - and $d$-type pairs have no critical value. It is interesting that for $|a|>0.5 d$-type pairs have no threshold to bind unlike the $s^{*}$-type pairs for $U=0$.

In conclusion we have shown that nnn hopping increases the pair mobility, which can be large even in the case of strong binding, strongly favoring the $d$-type pairing. We have shown a possibility of existence of a $s^{*}-d$ mixed state in certain areas of the $\mathrm{Bz}$. Our results for the $s^{*}$-wave pairs are directly related to the stability of $s^{*}$-wave superconductivity in a dilute limit on 2D lattice [3].

\section{Acknowledgments}

This work was supported by the Committee for Scientific Research grant No. 2PO3B05614.

\section{References}

[1] A.S. Blaer, H.C. Ren, O. Tchernyshyov, Phys. Rev. B 55, 6035 (1997).

[2] R. Micnas, J. Ranniger, S. Robaszkiewicz, Rev. Mod. Phys. 62, 113 (1990).

[3] M. Randeria, J.-M. Duan, L.-Y. Shieh, Phys. Rev. Lett. 62, 981 (1989). 